\title{
On the Spectrum of a Discrete Non-Hermitian Quantum System ${ }^{\star}$
}

\author{
Ebru ERGUN
}

Department of Physics, Ankara University, 06100 Tandogan, Ankara, Turkey

E-mail: eergun@science.ankara.edu.tr

Received October 28, 2008, in final form January 13, 2009; Published online January 19, 2009

doi:10.3842/SIGMA.2009.007

\begin{abstract}
In this paper, we develop spectral analysis of a discrete non-Hermitian quantum system that is a discrete counterpart of some continuous quantum systems on a complex contour. In particular, simple conditions for discreteness of the spectrum are established.
\end{abstract}

Key words: difference operator; non-Hermiticity; spectrum; eigenvalue; eigenvector; completely continuous operator

2000 Mathematics Subject Classification: 39A70; 81Q10

\section{Introduction}

Non-Hermitian Hamiltonians and complex extension of quantum mechanics have recently received a lot of attention (see review [1]). This field of mathematical physics came into flower due to the eigenvalue problem

$$
-\psi^{\prime \prime}(x)+i x^{3} \psi(x)=E \psi(x), \quad-\infty<x<\infty,
$$

where $\psi(x)$ is a desired solution, $i=\sqrt{-1}$ is the imaginary unit, $E$ is a complex parameter ("energy" or spectral parameter).

A complex value $E_{0}$ of the parameter $E$ is called an eigenvalue of equation (1.1) if this equation with $E=E_{0}$ has a nontrivial (non-identically zero) solution $\psi_{0}(x)$ that belongs to the Hilbert space $L^{2}(-\infty, \infty)$ of complex-valued functions $f$ defined on $(-\infty, \infty)$ such that

$$
\int_{-\infty}^{\infty}|f(x)|^{2} d x<\infty
$$

with the inner (scalar) product

$$
\langle f, g\rangle=\int_{-\infty}^{\infty} f(x) \bar{g}(x) d x
$$

in which the bar over a function denotes the complex conjugate. The function $\psi_{0}(x)$ is called an eigenfunction of equation (1.1), corresponding to the eigenvalue $E_{0}$.

If we define the operator $S: D \subset L^{2}(-\infty, \infty) \rightarrow L^{2}(-\infty, \infty)$ with the domain $D$ consisting of the functions $f \in L^{2}(-\infty, \infty)$ that are differentiable, with the derivative $f^{\prime}$ absolutely continuous on each finite subinterval of $(-\infty, \infty)$ and such that

$$
-f^{\prime \prime}+i x^{3} f \in L^{2}(-\infty, \infty)
$$

*This paper is a contribution to the Proceedings of the VIIth Workshop "Quantum Physics with NonHermitian Operators" (June 29 - July 11, 2008, Benasque, Spain). The full collection is available at http://www.emis.de/journals/SIGMA/PHHQP2008.html 
by letting

$$
S f=-f^{\prime \prime}+i x^{3} f \quad \text { for } \quad f \in D \text {, }
$$

then eigenvalue problem (1.1) can be written as $S \psi=E \psi, \psi \in D, \psi \neq 0$. Consequently, eigenvalues of equation (1.1) are eigenvalues of the operator $S$.

Because of the fact that the operator $S$ defined by (1.3) has the complex non-real coefficient (potential) equal to $i x^{3}$, this operator is not Hermitian with respect to the inner product (1.2), that is, we cannot state that

$$
\langle S f, g\rangle=\langle f, S g\rangle \quad \text { for all } f, g \in D \text {. }
$$

Therefore it is not obvious that the eigenvalues of $S$ may be real (remember that the eigenvalues of any Hermitian operator are real and the eigenvectors corresponding to the distinct eigenvalues are orthogonal).

Around 1992 Bessis and Zinn-Justin had noticed on the basis of numerical work that some of the eigenvalues of equation (1.1) seemed to be real and positive and they conjectured (not in print) that for equation (1.1) the eigenvalues are all real and positive.

In 1998, Bender and Boettcher [2] generalized the BZJ conjecture, namely, they conjectured (again on the basis of numerical analysis) that the eigenvalues of the equation

$$
-\psi^{\prime \prime}(x)-(i x)^{m} \psi(x)=E \psi(x), \quad-\infty<x<\infty,
$$

are all real and positive provided $m \geq 2$. Note that in equation (1.4) $m$ is an arbitrary positive real number and equation (1.1) corresponds to the choice $m=3$ in (1.4).

Bender and Boettcher assumed that the reason for reality of eigenvalues of (1.4) (in particular of (1.1)) must be certain symmetry property of this equation, namely, the so-called $P T$-symmetry of it (for more details see [1]).

$P$ (parity) and $T$ (time reversal) operations are defined by

$$
P f(x)=f(-x) \quad \text { and } \quad T f(x)=\bar{f}(x),
$$

respectively. A Hamiltonian

$$
H=-\frac{d^{2}}{d x^{2}}+V(x)
$$

with complex potential $V(x)$ is called $P T$-symmetric if it commutes with the composite operation PT:

$$
[H, P T]=H P T-P T H=0 .
$$

It is easily seen that $P T$-symmetricity of $H$ given by (1.5) is equivalent to the condition

$$
\bar{V}(-x)=V(x) .
$$

The potentials $i x^{3}$ and $-(i x)^{m}$ of equations (1.1) and (1.4), respectively, are PT-symmetric (note that $i x^{3}$ is not $P$-symmetric and $T$-symmetric, separately).

The first rigorous proof of reality and positivity of the eigenvalues of equation (1.4) was given in 2001 by Dorey, Dunning, and Tateo [3] (see also [4]).

Note that for $0<m<2$ the spectrum of (1.4) considered in $L^{2}(-\infty, \infty)$ is also discrete, however in this case only a finite number of eigenvalues are real and positive, and remaining eigenvalues (they are of infinite number) are non-real. Besides, if $m=4 k(k=1,2, \ldots)$ then for any complex value of $E$ all solutions of equation (1.4) belong to $L^{2}(-\infty, \infty)$ (the so-called Weyl's 
limit-circle case holds) and, therefore, all complex values of $E$ are eigenvalues of equation (1.4) and hence the spectrum is not discrete for $m=4 k(k=1,2, \ldots)$ if we consider the problem in $L^{2}(-\infty, \infty)$. In order to have in the case $m \geq 4$ a problem with discrete spectrum Bender and Boettcher made in [2] an important observation that the equation can be considered on an appropriately chosen complex contour. Namely, it is sufficient to consider the equation

$$
-\psi^{\prime \prime}(z)-(i z)^{m} \psi(z)=E \psi(z), \quad z \in \Gamma
$$

together with the condition that

$$
|\psi(z)| \rightarrow 0 \text { exponentially as } z \text { moves off to infinity along } \Gamma \text {, }
$$

where $\Gamma$ is a contour of the form

$$
\Gamma=\{z=x-i|x| \tan \delta:-\infty<x<\infty\}
$$

with

$$
\delta=\frac{\pi(m-2)}{2(m+2)} .
$$

Note that the contour $\Gamma$ forms an angle in the lower complex $z$-plane, of value $\pi-2 \delta$ with the vertex at the origin and symmetric with respect to the imaginary axis.

Next, Mostafazadeh showed in [5] that problem (1.6), (1.7) is equivalent to finding solutions in $L^{2}(-\infty, \infty)$ of the problem

$$
\begin{aligned}
& -\psi^{\prime \prime}(x)+|x|^{m} \psi(x)=E \rho(x) \psi(x), \quad x \in(-\infty, 0) \cup(0, \infty), \\
& \psi\left(0^{-}\right)=\psi\left(0^{+}\right), \quad \psi^{\prime}\left(0^{-}\right)=e^{2 i \delta} \psi^{\prime}\left(0^{+}\right),
\end{aligned}
$$

where

$$
\rho(x)= \begin{cases}e^{2 i \delta} & \text { if } \quad x<0 \\ e^{-2 i \delta} & \text { if } \quad x>0\end{cases}
$$

The main distinguishing features of problem (1.8), (1.9) are that it involves a complex-valued coefficient function $\rho(x)$ of the form (1.10) and that transition conditions (impulse conditions) of the form (1.9) are presented which also involve a complex coefficient. Such a problem is non-Hermitian with respect to the usual inner product $(1.2)$ of space $L^{2}(-\infty, \infty)$.

Our aim in this paper is to construct and investigate a discrete version of problem (1.8), (1.9). Discrete equations (difference equations) form a reach field, both interesting and useful [6, 7]. Discrete equations arise when differential equations are solved approximately by discretization. On the other hand they often arise independently as mathematical models of many practical events. Discrete equations can easily been algorithmized to solve them on computers. There is only a small body of work concerning discrete non-Hermitian quantum systems. Some examples are $[8,9,10,11,12,13,14,15]$. Note that in [15] the author considered a finite discrete interval version of the infinite discrete interval problem (1.12), (1.13) formulated below, and found conditions that ensure the reality of the eigenvalues. In the finite discrete interval case with the zero boundary conditions the problem is reduced to the eigenvalue problem for a finitedimensional tridiagonal matrix.

Let $\mathbb{Z}$ denote the set of all integers. For any $l, m \in \mathbb{Z}$ with $l \leq m,[l, m]$ will denote the discrete interval being the set $\{l, l+1, \ldots, m\}$. Semi-infinite intervals of the form $(-\infty, l]$ and $[l, \infty)$ will denote the discrete sets $\{\ldots, l-2, l-1, l\}$ and $\{l, l+1, l+2, \ldots\}$, respectively. Throughout the paper all intervals will be discrete intervals. Let us set

$$
\mathbb{Z}_{0}=\mathbb{Z} \backslash\{0,1\}=\{\ldots,-3,-2,-1\} \cup\{2,3,4, \ldots\}=(-\infty,-1] \cup[2, \infty) .
$$


We offer a discrete version of problem (1.8), (1.9) to be

$$
\begin{aligned}
& -\Delta^{2} y_{n-1}+q_{n} y_{n}=\lambda \rho_{n} y_{n}, \quad n \in \mathbb{Z}_{0}, \\
& y_{-1}=y_{1}, \quad \Delta y_{-1}=e^{2 i \delta} \Delta y_{1},
\end{aligned}
$$

where $y=\left(y_{n}\right)_{n \in \mathbb{Z}}$ is a desired solution, $\Delta$ is the forward difference operator defined by $\Delta y_{n}=$ $y_{n+1}-y_{n}$ so that $\Delta^{2} y_{n-1}=y_{n+1}-2 y_{n}+y_{n-1}$, the coefficients $q_{n}$ are real numbers given for $n \in \mathbb{Z}_{0}, \delta \in[0, \pi / 2)$, and $\rho_{n}$ are given for $n \in \mathbb{Z}_{0}$ by

$$
\rho_{n}=\left\{\begin{array}{lll}
e^{2 i \delta} & \text { if } \quad n \leq-1 \\
e^{-2 i \delta} & \text { if } \quad n \geq 2
\end{array}\right.
$$

One of the main results of the present paper is that if

$$
q_{n} \geq c>0 \quad \text { for } n \in \mathbb{Z}_{0}
$$

and

$$
\lim _{|n| \rightarrow \infty} q_{n}=\infty
$$

then the spectrum of problem (1.12), (1.13) is discrete.

The paper is organized as follows. In Section 2, we choose a suitable Hilbert space and define the main linear operators $L, M$, and $A=M^{-1} L$ related to problem (1.12), (1.13). Using these operators we introduce the concept of the spectrum for problem (1.12), (1.13). In Section 3, we demonstrate non-Hermiticity of the operators $L$ and $A$. In Section 4 , we present general properties of solutions of equations of type (1.12), (1.13). In Section 5, we construct two special solutions of problem (1.12), (1.13) under the condition (1.15). Using these solutions we show in Section 6 that the operator $L$ is invertible and we describe the structure of the inverse operator $L^{-1}$. Finally, in Section 7, we show that the operator $L^{-1}$ is completely continuous if, in addition, the condition (1.16) is satisfied. This fact yields the discreteness of the spectrum of problem (1.12), (1.13).

\section{The concept of the spectrum for problem (1.12), (1.13)}

In order to introduce the concept of the spectrum for problem (1.12), (1.13), define the Hilbert space $l_{0}^{2}$ of complex sequences $y=\left(y_{n}\right)_{n \in \mathbb{Z}_{0}}$ such that

$$
\sum_{n \in \mathbb{Z}_{0}}\left|y_{n}\right|^{2}<\infty
$$

with the inner product and norm

$$
\langle y, z\rangle=\sum_{n \in \mathbb{Z}_{0}} y_{n} \bar{z}_{n}, \quad\|y\|=\sqrt{\langle y, y\rangle}=\left\{\sum_{n \in \mathbb{Z}_{0}}\left|y_{n}\right|^{2}\right\}^{1 / 2},
$$

where $\mathbb{Z}_{0}$ is defined by (1.11) and the bar over a complex number denotes the complex conjugate.

Now we try to rewrite problem (1.12), (1.13) in the form of an equivalent vector equation in $l_{0}^{2}$ using appropriate operators. Denote by $D$ the linear set of all vectors $y=\left(y_{n}\right)_{n \in \mathbb{Z}_{0}} \in l_{0}^{2}$ such that $\left(q_{n} y_{n}\right)_{n \in \mathbb{Z}_{0}} \in l_{0}^{2}$. Taking this set as the domain of $L, L: D \subset l_{0}^{2} \rightarrow l_{0}^{2}$ is defined by

$$
(L y)_{n}=-\Delta^{2} y_{n-1}+q_{n} y_{n} \quad \text { for } n \in \mathbb{Z}_{0},
$$


where $y_{0}$ and $y_{1}$ are defined from the equations

$$
y_{-1}=y_{1}, \quad \Delta y_{-1}=e^{2 i \delta} \Delta y_{1} .
$$

Note that $y_{0}$ and $y_{1}$ are needed when we evaluate $(L y)_{n}$ for $n=-1$ and $n=2$, respectively:

$$
\begin{aligned}
& (L y)_{-1}=-\Delta^{2} y_{-2}+q_{-1} y_{-1}=\left(y_{0}-2 y_{-1}+y_{-2}\right)+q_{-1} y_{-1}, \\
& (L y)_{2}=-\Delta^{2} y_{1}+q_{2} y_{2}=\left(y_{3}-2 y_{2}+y_{1}\right)+q_{2} y_{2}
\end{aligned}
$$

and (1.13) gives for $y_{1}, y_{0}$ the expressions

$$
\begin{aligned}
& y_{1}=y_{-1}, \\
& y_{0}=y_{-1}+e^{2 i \delta}\left(y_{2}-y_{1}\right)=\left(1-e^{2 i \delta}\right) y_{-1}+e^{2 i \delta} y_{2} .
\end{aligned}
$$

Next, define the operator $M: l_{0}^{2} \rightarrow l_{0}^{2}$ by

$$
(M y)_{n}=\rho_{n} y_{n} \quad \text { for } n \in \mathbb{Z}_{0},
$$

where $\rho_{n}$ is given by (1.14). Obviously, the adjoint $M^{*}$ of $M$ is defined by

$$
\left(M^{*} y\right)_{n}=\bar{\rho}_{n} y_{n}, \quad n \in \mathbb{Z}_{0},
$$

and since $\left|\rho_{n}\right|=1$, we get that $M$ is a unitary operator:

$$
M M^{*}=M^{*} M=I,
$$

where the asterisk denotes the adjoint operator and $I$ is the identity operator.

Therefore problem (1.12), (1.13) can be written as

$$
L y=\lambda M y, \quad y \in D, \quad \text { or } \quad M^{-1} L y=\lambda y, \quad y \in D .
$$

This motivates to introduce the following definition.

Definition 1. By the spectrum of problem (1.12), (1.13) is meant the spectrum of the operator $A=M^{-1} L$ with the domain $D$ in the space $l_{0}^{2}$.

Remember that (see [16]) if $A$ is a linear operator with a domain dense in a Hilbert space, then a complex number $\lambda$ is called a regular point of the operator $A$ if the inverse $(A-\lambda I)^{-1}$ exists and represents a bounded operator defined on the whole space. All other points of the complex plane comprise the spectrum of the operator $A$. Obviously the eigenvalues $\lambda$ of an operator belong to its spectrum, since the operator $(A-\lambda I)^{-1}$ does not exist for such points (the operator $A-\lambda I$ is not one-to-one). The set of all eigenvalues is called the point spectrum of the operator. The spectrum of the operator $A$ is said to be discrete if it consists of a denumerable (i.e., at most countable) set of eigenvalues with no finite point of accumulation.

A linear operator acting in a Hilbert space and defined on the whole space is called completely continuous if it maps bounded sets into relatively compact sets (a set is called relatively compact if every infinite subset of this set has a limit point in the space, that may not belong to the set). Any completely continuous operator is bounded and hence its spectrum is a compact subset of the complex plane. As is well known [16], every nonzero point of the spectrum of a completely continuous operator is an eigenvalue of finite multiplicity (that is, to each eigenvalue there correspond only a finite number of linearly independent eigenvectors); the set of eigenvalues is at most countable and can have only one accumulation point $\lambda=0$. It follows that if a linear operator $A$ with a domain dense in a Hilbert space is invertible and its inverse $A^{-1}$ is completely continuous, then the spectrum of $A$ is discrete.

In this paper we show that the operator $L$ is invertible under the condition (1.15) and that its inverse $L^{-1}$ is a completely continuous operator if, in addition, the condition (1.16) is satisfied. This implies that the operator $A=M^{-1} L$ is invertible and $A^{-1}=L^{-1} M$ is a completely continuous operator. Hence the spectrum of the operator $A$ is discrete. 


\section{Non-Hermiticity of the operators $L$ and $A$}

Let $\left(f_{k}\right)$ be a given complex sequence, where $k \in \mathbb{Z}$. The forward and backward difference operators $\Delta$ and $\nabla$ are defined by

$$
\Delta f_{k}=f_{k+1}-f_{k} \quad \text { and } \quad \nabla f_{k}=f_{k}-f_{k-1},
$$

respectively. We easily see that

$$
\begin{aligned}
& \nabla f_{k}=\Delta f_{k-1}, \\
& \Delta^{2} f_{k}=\Delta\left(\Delta f_{k}\right)=f_{k+2}-2 f_{k+1}+f_{k}, \\
& \nabla^{2} f_{k}=\nabla\left(\nabla f_{k}\right)=f_{k}-2 f_{k-1}+f_{k-2}, \\
& \Delta \nabla f_{k}=f_{k+1}-2 f_{k}+f_{k-1}=\nabla \Delta f_{k}=\Delta^{2} f_{k-1}=\nabla^{2} f_{k+1} .
\end{aligned}
$$

For any integers $a, b \in \mathbb{Z}$ with $a<b$ we have the summation by parts formulas

$$
\begin{aligned}
& \sum_{k=a}^{b}\left(\Delta f_{k}\right) g_{k}=\left.f_{k+1} g_{k}\right|_{a-1} ^{b}-\sum_{k=a}^{b} f_{k}\left(\nabla g_{k}\right)=f_{b+1} g_{b}-f_{a} g_{a-1}-\sum_{k=a}^{b} f_{k}\left(\nabla g_{k}\right), \\
& \sum_{k=a}^{b}\left(\nabla f_{k}\right) g_{k}=\left.f_{k} g_{k+1}\right|_{a-1} ^{b}-\sum_{k=a}^{b} f_{k}\left(\Delta g_{k}\right)=f_{b} g_{b+1}-f_{a-1} g_{a}-\sum_{k=a}^{b} f_{k}\left(\Delta g_{k}\right), \\
& \sum_{k=a}^{b}\left(\Delta \nabla f_{k}\right) g_{k}=\left.\left(\Delta f_{k}\right) g_{k}\right|_{a-1} ^{b}-\sum_{k=a}^{b}\left(\nabla f_{k}\right)\left(\nabla g_{k}\right), \\
& \sum_{k=a}^{b}\left(\Delta \nabla f_{k}\right) g_{k}=\left.\left(\Delta f_{k}\right) g_{k+1}\right|_{a-1} ^{b}-\sum_{k=a}^{b}\left(\Delta f_{k}\right)\left(\Delta g_{k}\right), \\
& \sum_{k=a}^{b}\left[\left(\Delta \nabla f_{k}\right) g_{k}-f_{k}\left(\Delta \nabla g_{k}\right)\right]=\left[\left(\Delta f_{k}\right) g_{k}-f_{k}\left(\Delta g_{k}\right)\right]_{a-1}^{b} \\
& =\left[\left(\Delta f_{b}\right) g_{b}-f_{b}\left(\Delta g_{b}\right)\right]-\left[\left(\Delta f_{a-1}\right) g_{a-1}-f_{a-1}\left(\Delta g_{a-1}\right)\right] .
\end{aligned}
$$

Theorem 1. Let $\delta \in[0, \pi / 2)$. If $\delta=0$, then the operator $L$ is Hermitian:

$$
\langle L y, z\rangle=\langle y, L z\rangle \quad \text { for all } y, z \in D \text {. }
$$

But if $\delta \neq 0$, then the operator $L$ is not Hermitian.

Proof. Using formula (3.3) and equation

$$
(L y)_{n}=-\Delta^{2} y_{n-1}+q_{n} y_{n}=-\Delta \nabla y_{n}+q_{n} y_{n} \quad \text { for } n \in \mathbb{Z}_{0},
$$

where $y_{0}$ and $y_{1}$ are defined from the equations

$$
y_{-1}=y_{1}, \quad \Delta y_{-1}=e^{2 i \delta} \Delta y_{1},
$$

and taking into account that for any $y=\left(y_{n}\right)_{n \in \mathbb{Z}_{0}} \in l_{0}^{2}$ we have $y_{n} \rightarrow 0, \Delta y_{n} \rightarrow 0$ as $|n| \rightarrow \infty$, we get for all $y, z \in D$,

$$
\begin{aligned}
\langle L y, z\rangle-\langle y, L z\rangle & =-\sum_{n \in \mathbb{Z}_{0}}\left[\left(\Delta \nabla y_{n}\right) \bar{z}_{n}-y_{n}\left(\Delta \nabla \bar{z}_{n}\right)\right] \\
& =-\sum_{-\infty}^{n=-1}\left[\left(\Delta \nabla y_{n}\right) \bar{z}_{n}-y_{n}\left(\Delta \nabla \bar{z}_{n}\right)\right]-\sum_{n=2}^{\infty}\left[\left(\Delta \nabla y_{n}\right) \bar{z}_{n}-y_{n}\left(\Delta \nabla \bar{z}_{n}\right)\right]
\end{aligned}
$$




$$
\begin{aligned}
& =-\left[\left(\Delta y_{-1}\right) \bar{z}_{-1}-y_{-1}\left(\Delta \bar{z}_{-1}\right)\right]+\left[\left(\Delta y_{1}\right) \bar{z}_{1}-y_{1}\left(\Delta \bar{z}_{1}\right)\right] \\
& =-\left[\left(\Delta y_{-1}\right) \bar{z}_{-1}-y_{-1}\left(\Delta \bar{z}_{-1}\right)\right]+\left[e^{-2 i \delta}\left(\Delta y_{-1}\right) \bar{z}_{-1}-y_{-1} e^{2 i \delta}\left(\Delta \bar{z}_{-1}\right)\right] \\
& =\left(e^{-2 i \delta}-1\right)\left(\Delta y_{-1}\right) \bar{z}_{-1}-\left(e^{2 i \delta}-1\right) y_{-1}\left(\Delta \bar{z}_{-1}\right) .
\end{aligned}
$$

Thus,

$$
\langle L y, z\rangle-\langle y, L z\rangle=\left(e^{-2 i \delta}-1\right)\left(\Delta y_{-1}\right) \bar{z}_{-1}-\left(e^{2 i \delta}-1\right) y_{-1}\left(\Delta \bar{z}_{-1}\right),
$$

for all $y, z \in D$. Formula (3.5) shows that if $\delta=0$, then the operator $L$ is Hermitian:

$$
\langle L y, z\rangle=\langle y, L z\rangle \quad \text { for all } y, z \in D .
$$

The same formula shows that if $\delta \neq 0$ (recall that $\delta \in[0, \pi / 2)$ ), then the operator $L$ is not Hermitian:

$$
\langle L y, z\rangle \neq\langle y, L z\rangle \quad \text { for some } y, z \in D .
$$

The theorem is proved.

Theorem 2. Let $\delta \in[0, \pi / 2)$. If $\delta=0$, then the operator $A=M^{-1} L$ is Hermitian:

$$
\langle A y, z\rangle=\langle y, A z\rangle \quad \text { for all } y, z \in D .
$$

But if $\delta \neq 0$, then the operator $A$ is not Hermitian.

Proof. We have for any $y, z \in D$,

$$
\begin{aligned}
\langle A y, z\rangle-\langle y, A z\rangle & =\left\langle M^{-1} L y, z\right\rangle-\left\langle y, M^{-1} L z\right\rangle=\langle L y, M z\rangle-\langle M y, L z\rangle \\
& =\sum_{n \in \mathbb{Z}_{0}}\left[-\left(\Delta \nabla y_{n}\right)+q_{n} y_{n}\right] \overline{\rho_{n} z_{n}}-\sum_{n \in \mathbb{Z}_{0}} \rho_{n} y_{n}\left[-\left(\Delta \nabla \bar{z}_{n}\right)+q_{n} \bar{z}_{n}\right] \\
& =-\sum_{n \in \mathbb{Z}_{0}}\left[\left(\Delta \nabla y_{n}\right) \overline{\rho_{n} z_{n}}-\rho_{n} y_{n}\left(\Delta \nabla \bar{z}_{n}\right)\right]+\sum_{n \in \mathbb{Z}_{0}}\left(\bar{\rho}_{n}-\rho_{n}\right) q_{n} y_{n} \bar{z}_{n} .
\end{aligned}
$$

Next, from

$$
\rho_{n}=\left\{\begin{array}{ll}
e^{2 i \delta} & \text { if } n \leq-1, \\
e^{-2 i \delta} & \text { if } n \geq 2,
\end{array} \quad \text { and } \quad \bar{\rho}_{n}=\left\{\begin{array}{lll}
e^{-2 i \delta} & \text { if } n \leq-1, \\
e^{2 i \delta} & \text { if } n \geq 2,
\end{array}\right.\right.
$$

we find

$$
\bar{\rho}_{n}-\rho_{n}= \begin{cases}-2 i \sin 2 \delta & \text { if } \quad n \leq-1, \\ 2 i \sin 2 \delta & \text { if } \quad n \geq 2\end{cases}
$$

so that

$$
\sum_{n \in \mathbb{Z}_{0}}\left(\bar{\rho}_{n}-\rho_{n}\right) q_{n} y_{n} \bar{z}_{n}=-2 i \sin 2 \delta \sum_{-\infty}^{n=-1} q_{n} y_{n} \bar{z}_{n}+2 i \sin 2 \delta \sum_{n=2}^{\infty} q_{n} y_{n} \bar{z}_{n} .
$$

Besides, using formula (3.2) and equations in (3.4), we obtain

$$
\begin{gathered}
-\sum_{n \in \mathbb{Z}_{0}}\left[\left(\Delta \nabla y_{n}\right) \overline{\rho_{n} z_{n}}-\rho_{n} y_{n}\left(\Delta \nabla \bar{z}_{n}\right)\right]=-e^{-2 i \delta} \sum_{-\infty}^{n=-1}\left(\Delta \nabla y_{n}\right) \bar{z}_{n}-e^{2 i \delta} \sum_{n=2}^{\infty}\left(\Delta \nabla y_{n}\right) \bar{z}_{n} \\
+e^{2 i \delta} \sum_{-\infty}^{n=-1} y_{n}\left(\Delta \nabla \bar{z}_{n}\right)+e^{-2 i \delta} \sum_{n=2}^{\infty} y_{n}\left(\Delta \nabla \bar{z}_{n}\right)
\end{gathered}
$$




$$
\begin{aligned}
= & -e^{-2 i \delta}\left(\Delta y_{-1}\right) \bar{z}_{-1}+e^{-2 i \delta} \sum_{-\infty}^{n=-1}\left(\nabla y_{n}\right)\left(\nabla \bar{z}_{n}\right)+e^{2 i \delta}\left(\Delta y_{1}\right) \bar{z}_{1}+e^{2 i \delta} \sum_{n=2}^{\infty}\left(\nabla y_{n}\right)\left(\nabla \bar{z}_{n}\right) \\
& +e^{2 i \delta} y_{-1}\left(\Delta \bar{z}_{-1}\right)-e^{2 i \delta} \sum_{-\infty}^{n=-1}\left(\nabla y_{n}\right)\left(\nabla \bar{z}_{n}\right)-e^{-2 i \delta} y_{1}\left(\Delta \bar{z}_{1}\right)-e^{-2 i \delta} \sum_{n=2}^{\infty}\left(\nabla y_{n}\right)\left(\nabla \bar{z}_{n}\right) \\
= & \left(1-e^{-2 i \delta}\right)\left(\Delta y_{-1}\right) \bar{z}_{-1}-\left(1-e^{2 i \delta}\right) y_{-1}\left(\Delta \bar{z}_{-1}\right) \\
& -2 i \sin 2 \delta \sum_{-\infty}^{n=-1}\left(\nabla y_{n}\right)\left(\nabla \bar{z}_{n}\right)+2 i \sin 2 \delta \sum_{n=2}^{\infty}\left(\nabla y_{n}\right)\left(\nabla \bar{z}_{n}\right) .
\end{aligned}
$$

Thus,

$$
\begin{aligned}
& \langle A y, z\rangle-\langle y, A z\rangle=\left(1-e^{-2 i \delta}\right)\left(\Delta y_{-1}\right) \bar{z}_{-1}-\left(1-e^{2 i \delta}\right) y_{-1}\left(\Delta \bar{z}_{-1}\right) \\
& -2 i \sin 2 \delta \sum_{-\infty}^{n=-1}\left[\left(\nabla y_{n}\right)\left(\nabla \bar{z}_{n}\right)+q_{n} y_{n} \bar{z}_{n}\right]+2 i \sin 2 \delta \sum_{n=2}^{\infty}\left[\left(\nabla y_{n}\right)\left(\nabla \bar{z}_{n}\right)+q_{n} y_{n} \bar{z}_{n}\right] .
\end{aligned}
$$

Formula (3.6) shows that if $\delta=0$, then the operator $A$ is Hermitian and if $\delta \neq 0$, then $A$ is not Hermitian.

Remark 1. In the case $\delta=0$ we have $A=L$.

\section{Second order linear difference equations with impulse}

Consider the second order linear homogeneous difference equation with impulse

$$
\begin{aligned}
& -\Delta^{2} y_{n-1}+p_{n} y_{n}=0, \quad n \in \mathbb{Z}_{0}=\mathbb{Z} \backslash\{0,1\}=(-\infty,-1] \cup[2, \infty), \\
& y_{-1}=d_{1} y_{1}, \quad \Delta y_{-1}=d_{2} \Delta y_{1},
\end{aligned}
$$

where $y=\left(y_{n}\right)$ with $n \in \mathbb{Z}$ is a desired solution, the coefficients $p_{n}$ are complex numbers given for $n \in \mathbb{Z}_{0} ; d_{1}, d_{2}$ presented in the "impulse conditions" (transition conditions) in (4.2) are nonzero complex numbers.

Using the definition of $\Delta$-derivative we can rewrite problem (4.1), (4.2) in the form

$$
\begin{aligned}
& -y_{n-1}+\widetilde{p}_{n} y_{n}-y_{n+1}=0, \quad n \in(-\infty,-1] \cup[2, \infty), \\
& y_{-1}=d_{1} y_{1}, \quad y_{0}-y_{-1}=d_{2}\left(y_{2}-y_{1}\right),
\end{aligned}
$$

where

$$
\widetilde{p}_{n}=p_{n}+2, \quad n \in(-\infty,-1] \cup[2, \infty) .
$$

Theorem 3. Let $n_{0}$ be a fixed point in $\mathbb{Z}$ and $c_{0}, c_{1}$ be given complex numbers. Then problem (4.1), (4.2) has a unique solution $\left(y_{n}\right), n \in \mathbb{Z}$, such that

$$
y_{n_{0}}=c_{0}, \quad \Delta y_{n_{0}}=c_{1}, \quad \text { that is, } \quad y_{n_{0}}=c_{0}, \quad y_{n_{0}+1}=c_{0}+c_{1}=c_{1}^{\prime} .
$$

Proof. First assume that $n_{0} \in(-\infty,-1]$. We can rewrite equation (4.3) in the form

$$
y_{n-1}=\widetilde{p}_{n} y_{n}-y_{n+1}=0, \quad n \in(-\infty,-1] \cup[2, \infty)
$$

as well as in the form

$$
y_{n+1}=\widetilde{p}_{n} y_{n}-y_{n-1}=0, \quad n \in(-\infty,-1] \cup[2, \infty) .
$$


Using the initial conditions (4.5) we find, recurrently (step by step), $y_{n}$ for $n \leq n_{0}+1$ uniquely from (4.6) and for $n_{0}+2 \leq n \leq-1$ uniquely from (4.7). Then we find $y_{1}$ and $y_{2}$ from the transition conditions (4.4) and then we find $y_{n}$ for $n \geq 3$ uniquely from (4.7).

In the case $n_{0} \in[1, \infty)$ we are reasoning similarly; using equations (4.6), (4.7) we first find $y_{n}$ uniquely for $n \geq 1$ and then using the transition conditions (4.4) we pass to the interval $(-\infty,-1]$.

Finally, if $n_{0}=0$, then we find $y_{0}$ and $y_{1}$ uniquely from the initial conditions (4.5) with $n_{0}=0$. Then we find $y_{-1}$ and $y_{2}$ from the transition conditions (4.4). Next, solving equation (4.6) at first on $(-\infty,-1]$ we find $y_{n}$ uniquely for $n \in(-\infty,-2]$ and then solving $(4.7)$ on $[2, \infty)$ we find $y_{n}$ uniquely for $n \in[3, \infty)$.

Definition 2. For two sequences $y=\left(y_{n}\right)$ and $z=\left(z_{n}\right)$ with $n \in \mathbb{Z}$, we define their Wronskian by

$$
W_{n}(y, z)=y_{n} \Delta z_{n}-\left(\Delta y_{n}\right) z_{n}=y_{n} z_{n+1}-y_{n+1} z_{n}, \quad n \in \mathbb{Z} .
$$

Theorem 4. The Wronskian of any two solutions $y$ and $z$ of problem (4.1), (4.2) is constant on each of the intervals $(-\infty,-1]$ and $[1, \infty)$ :

$$
W_{n}(y, z)=\left\{\begin{array}{lll}
\omega^{-} & \text {if } & n \in(-\infty,-1] \\
\omega^{+} & \text {if } n \in[1, \infty)
\end{array}\right.
$$

In addition,

$$
\omega^{-}=d_{1} d_{2} \omega^{+}
$$

and

$$
W_{0}(y, z)=-d_{2} \omega^{+} .
$$

Proof. Suppose that $y=\left(y_{n}\right)$ and $z=\left(z_{n}\right)$, where $n \in \mathbb{Z}$, are solutions of (4.1), (4.2). Let us compute the $\Delta$-derivative of $W_{n}(y, z)$. Using the product rule for $\Delta$-derivative

$$
\Delta\left(f_{n} g_{n}\right)=\left(\Delta f_{n}\right) g_{n}+f_{n+1} \Delta g_{n}=f_{n} \Delta g_{n}+\left(\Delta f_{n}\right) g_{n+1},
$$

we have

$$
\begin{aligned}
\Delta W_{n}(y, z) & =\Delta\left[y_{n} \Delta z_{n}-\left(\Delta y_{n}\right) z_{n}\right]=\left(\Delta y_{n}\right) \Delta z_{n}+y_{n+1} \Delta^{2} z_{n}-\left(\Delta y_{n}\right) \Delta z_{n}-\left(\Delta^{2} y_{n}\right) z_{n+1} \\
& =y_{n+1} \Delta^{2} z_{n}-\left(\Delta^{2} y_{n}\right) z_{n+1} .
\end{aligned}
$$

Further, since $y_{n}$ and $z_{n}$ are solutions of (4.1), (4.2),

$$
\begin{array}{rlrl}
\Delta^{2} y_{n} & =p_{n+1} y_{n+1}, & & n \in(-\infty,-2] \cup[1, \infty), \\
\Delta^{2} z_{n}=p_{n+1} z_{n+1}, & & n \in(-\infty,-2] \cup[1, \infty) .
\end{array}
$$

Therefore

$$
\Delta W_{n}(y, z)=0 \quad \text { for } n \in(-\infty,-2] \cup[1, \infty) .
$$

The latter implies that $W_{n}(y, z)$ is constant on $(-\infty,-1]$ and on $[1, \infty)$. Thus we have (4.8), where $\omega^{-}$and $\omega^{+}$are some constants (depending on the solutions $y$ and $z$ ).

Next using (4.8) and the impulse conditions in (4.2) for $y_{n}$ and $z_{n}$, we have

$$
\begin{aligned}
\omega^{-} & =W_{-1}(y, z)=y_{-1} \Delta z_{-1}-\left(\Delta y_{-1}\right) z_{-1}=d_{1} d_{2}\left[y_{1} \Delta z_{1}-\left(\Delta y_{1}\right) z_{1}\right] \\
& =d_{1} d_{2} W_{1}(y, z)=d_{1} d_{2} \omega^{+}
\end{aligned}
$$

so that (4.9) is established. 
Finally, from the impulse conditions in (4.2) we find that

$$
y_{0}=\left(d_{1}-d_{2}\right) y_{1}+d_{2} y_{2} \text {. }
$$

Substituting this expression for $y_{0}$ and $z_{0}$ into

$$
W_{0}(y, z)=y_{0} z_{1}-y_{1} z_{0}
$$

we get

$$
W_{0}(y, z)=-d_{2} W_{1}(y, z)=-d_{2} \omega^{+} .
$$

Therefore (4.10) is also proved.

Corollary 1. If $y$ and $z$ are two solutions of (4.1), (4.2), then either $W_{n}(y, z)=0$ for all $n \in \mathbb{Z}$ or $W_{n}(y, z) \neq 0$ for all $n \in \mathbb{Z}$.

By using Theorem 3, the following two theorems can be proved in exactly the same way when equation (4.1) does not include any impulse conditions [6].

Theorem 5. Any two solutions of (4.1), (4.2) are linearly independent if and only if their Wronskian is not zero.

Theorem 6. Problem (4.1), (4.2) has two linearly independent solutions and every solution of (4.1), (4.2) is a linear combination of these solutions.

We say that $y=\left(y_{n}\right)$ and $z=\left(z_{n}\right)$, where $n \in \mathbb{Z}$, form a fundamental set (or fundamental system) of solutions for (4.1), (4.2) provided that they are solutions of (4.1), (4.2) and their Wronskian is not zero.

Let us consider the nonhomogeneous equation

$$
-\Delta^{2} y_{n-1}+p_{n} y_{n}=h_{n}, \quad n \in(-\infty,-1] \cup[2, \infty),
$$

with the impulse conditions

$$
y_{-1}=d_{1} y_{1}, \quad \Delta y_{-1}=d_{2} \Delta y_{1},
$$

where $h_{n}$ is a complex sequence defined for $n \in(-\infty,-1] \cup[2, \infty)$. We will extend $h_{n}$ to the values $n=0$ and $n=1$ by setting

$$
h_{0}=h_{1}=0 .
$$

Theorem 7. Suppose that $u=\left(u_{n}\right)$ and $v=\left(v_{n}\right)$ form a fundamental set of solutions of the homogeneous problem (4.1), (4.2). Then a general solution of the corresponding nonhomogeneous problem (4.11), (4.12) is given by

$$
y_{n}=c_{1} u_{n}+c_{2} v_{n}+x_{n}, \quad n \in \mathbb{Z},
$$

where $c_{1}, c_{2}$ are arbitrary constants and

$$
x_{n}= \begin{cases}-\sum_{s=n}^{0} \frac{u_{n} v_{s}-u_{s} v_{n}}{W_{s}(u, v)} h_{s} & \text { if } \quad n \leq 0, \\ \sum_{s=1}^{n} \frac{u_{n} v_{s}-u_{s} v_{n}}{W_{s}(u, v)} h_{s} & \text { if } \quad n \geq 1 .\end{cases}
$$

Proof. Taking into account (4.13) it is not difficult to verify that the sequence $x_{n}$ defined by (4.14) is a particular solution of (4.11), (4.12), namely, $x_{n}$ satisfies equation (4.11) and the conditions

$$
x_{-1}=\Delta x_{-1}=0, \quad x_{1}=\Delta x_{1}=0 .
$$

This implies that the statement of the theorem is true. 


\section{$5 \quad$ Two special solutions}

Consider the homogeneous problem

$$
\begin{aligned}
& -\Delta^{2} y_{n-1}+q_{n} y_{n}=0, \quad n \in \mathbb{Z}_{0}=\mathbb{Z} \backslash\{0,1\}, \\
& y_{-1}=y_{1}, \quad \Delta y_{-1}=e^{2 i \delta} \Delta y_{1},
\end{aligned}
$$

where $\delta \in[0, \pi / 2)$ is a fixed real number and

$$
q_{n} \geq c>0 \quad \text { for } \quad n \in \mathbb{Z}_{0} .
$$

In this section we show that under the condition (5.3) problem (5.1), (5.2) has two linearly independent solutions $\psi=\left(\psi_{n}\right)$ and $\chi=\left(\chi_{n}\right)$, where $n \in \mathbb{Z}$, such that

$$
\sum_{n=0}^{\infty}\left|\psi_{n}\right|^{2}<\infty \quad \text { and } \quad \sum_{-\infty}^{n=0}\left|\chi_{n}\right|^{2}<\infty
$$

These solutions will allow us to find the inverse $L^{-1}$ of the operator $L$ introduced above in Section 2 and investigate the properties of $L^{-1}$.

First we derive two simple useful formulas related to the nonhomogeneous problem

$$
\begin{aligned}
& -\Delta^{2} y_{n-1}+q_{n} y_{n}=f_{n}, \quad n \in \mathbb{Z}_{0}, \\
& y_{-1}=y_{1}, \quad \Delta y_{-1}=e^{2 i \delta} \Delta y_{1},
\end{aligned}
$$

where $\left(q_{n}\right)$ is a real sequence with $n \in \mathbb{Z}_{0}$, and $\delta \in[0, \pi / 2) ;\left(f_{n}\right)$ is a complex sequence with $n \in \mathbb{Z}_{0}$.

Lemma 1. Let $y=\left(y_{n}\right)$ with $n \in \mathbb{Z}$ be a solution of problem (5.5), (5.6) and $a, b$ be any integers such that $a \leq-1$ and $b \geq 2$. Then the following formulas hold:

$$
\begin{aligned}
& \sum_{n=2}^{b}\left(\left|\Delta y_{n}\right|^{2}+q_{n}\left|y_{n}\right|^{2}\right)=\left.\left(\Delta y_{n}\right) \bar{y}_{n+1}\right|_{1} ^{b}+\sum_{n=2}^{b} f_{n} \bar{y}_{n}, \\
& \sum_{n=a}^{-1}\left(\left|\Delta y_{n}\right|^{2}+q_{n}\left|y_{n}\right|^{2}\right)=\left.\left(\Delta y_{n}\right) \bar{y}_{n+1}\right|_{a-1} ^{-1}+\sum_{n=a}^{-1} f_{n} \bar{y}_{n} .
\end{aligned}
$$

Proof. To prove (5.7), multiply equation (5.5) by $\bar{y}_{n}$ and sum from $n=2$ to $n=b$ :

$$
-\sum_{n=2}^{b}\left(\Delta^{2} y_{n-1}\right) \bar{y}_{n}+\sum_{n=2}^{b} q_{n}\left|y_{n}\right|^{2}=\sum_{n=2}^{b} f_{n} \bar{y}_{n} .
$$

Next, applying the summation by parts formula (3.1) we get that

$$
-\sum_{n=2}^{b}\left(\Delta^{2} y_{n-1}\right) \bar{y}_{n}=-\sum_{n=2}^{b}\left(\nabla \Delta y_{n}\right) \bar{y}_{n}=-\left.\left(\Delta y_{n}\right) \bar{y}_{n+1}\right|_{1} ^{b}+\sum_{n=2}^{b}\left|\Delta y_{n}\right|^{2} .
$$

Therefore the formula (5.7) follows.

The formula (5.8) can be proved in a similar way.

Theorem 8. Under the condition (5.3) problem (5.1), (5.2) has two linearly independent solutions $\psi=\left(\psi_{n}\right)$ and $\chi=\left(\chi_{n}\right)$ with $n \in \mathbb{Z}$, possessing the properties stated in (5.4). 
Proof. Denote by $\varphi=\left(\varphi_{n}\right)$ and $\theta=\left(\theta_{n}\right)$, where $n \in \mathbb{Z}$, solutions of problem (5.1), (5.2) satisfying the initial conditions

$$
\begin{array}{ll}
\varphi_{1}=1, & \Delta \varphi_{1}=-1, \\
\theta_{1}=1, & \Delta \theta_{1}=0 .
\end{array}
$$

Such solutions exist and are unique by Theorem 3. It follows from (5.9), (5.10) that $\varphi_{2}=0$, $\theta_{2}=1$. According to Theorem 4 we find that

$$
W_{0}(\varphi, \theta)=-e^{2 i \delta}, \quad W_{n}(\varphi, \theta)=\left\{\begin{array}{lll}
e^{2 i \delta} & \text { if } & n \leq-1 \\
1 & \text { if } & n \geq 1
\end{array}\right.
$$

Therefore $W_{n}(\varphi, \theta) \neq 0$ and by Theorem 5 the solutions $\varphi$ and $\theta$ are linearly independent.

We seek the desired solution $\psi=\left(\psi_{n}\right)$ of problem (5.1), (5.2) in the form

$$
\psi_{n}=\varphi_{n}+v \theta_{n}, \quad n \in \mathbb{Z}
$$

where $v$ is a complex constant which we will choose.

Take an arbitrary integer $b \geq 2$. Applying (5.7) to

$$
\begin{aligned}
& -\Delta^{2} \psi_{n-1}+q_{n} \psi_{n}=0, \quad n \in \mathbb{Z}_{0}, \\
& \psi_{-1}=\psi_{1}, \quad \Delta \psi_{-1}=e^{2 i \delta} \Delta \psi_{1},
\end{aligned}
$$

we get

$$
\sum_{n=2}^{b}\left(\left|\Delta \psi_{n}\right|^{2}+q_{n}\left|\psi_{n}\right|^{2}\right)=\left.\left(\Delta \psi_{n}\right) \bar{\psi}_{n+1}\right|_{1} ^{b} .
$$

Since $\Delta \psi_{1}=-1$ and $\psi_{2}=v$, by (5.12) and (5.9), (5.10), hence

$$
\sum_{n=2}^{b}\left(\left|\Delta \psi_{n}\right|^{2}+q_{n}\left|\psi_{n}\right|^{2}\right)=\left(\Delta \psi_{b}\right) \bar{\psi}_{b+1}+\bar{v}
$$

Multiply the latter equality by $e^{i \delta}$ and take then the real part of both sides to get

$$
(\cos \delta) \sum_{n=2}^{b}\left(\left|\Delta \psi_{n}\right|^{2}+q_{n}\left|\psi_{n}\right|^{2}\right)=\operatorname{Re}\left\{e^{i \delta}\left(\Delta \psi_{b}\right) \bar{\psi}_{b+1}\right\}+\operatorname{Re}\left(v e^{-i \delta}\right) .
$$

Now we choose $v$ so that to have

$$
\operatorname{Re}\left\{e^{i \delta}\left(\Delta \psi_{b}\right) \bar{\psi}_{b+1}\right\}=0 .
$$

Since

$$
\operatorname{Re}\left\{e^{i \delta}\left(\Delta \psi_{b}\right) \bar{\psi}_{b+1}\right\}=\left|\psi_{b+1}\right|^{2} \operatorname{Re}\left\{e^{i \delta} \frac{\Delta \psi_{b}}{\psi_{b+1}}\right\},
$$

it is sufficient for (5.14) to have

$$
\operatorname{Re}\left\{e^{i \delta} \frac{\Delta \psi_{b}}{\psi_{b+1}}\right\}=0
$$

Note that $\psi_{b}$ cannot be zero for any two successive values of $b$ (otherwise $\psi_{n}$ would be identically zero by the uniqueness theorem for solution, that is not true since $\varphi$ and $\theta$ are linearly independent). Therefore $\psi_{b} \neq 0$ for infinitely many values of $b$. 
Under the condition (5.15) the equation (5.13) becomes

$$
(\cos \delta) \sum_{n=2}^{b}\left(\left|\Delta \psi_{n}\right|^{2}+q_{n}\left|\psi_{n}\right|^{2}\right)=\operatorname{Re}\left(v e^{-i \delta}\right) .
$$

The condition (5.15) can be written as

$$
e^{i \delta} \frac{\Delta \varphi_{b}+v \Delta \theta_{b}}{\varphi_{b+1}+v \theta_{b+1}}=\beta,
$$

where $\beta$ is a pure imaginary number $(\beta=i t, t \in \mathbb{R})$. Note that

$$
\varphi_{b+1} \Delta \theta_{b}-\left(\Delta \varphi_{b}\right) \theta_{b+1}=-\varphi_{b+1} \theta_{b}+\varphi_{b} \theta_{b+1}=W_{b}(\varphi, \theta)=1 \neq 0
$$

by (5.11). Therefore (5.16) defines a linear-fractional transformation of the complex $v$-plane onto the complex $\beta$-plane. Solving (5.16) for $v$, we get

$$
v(\beta)=\frac{\varphi_{b+1} \beta-e^{i \delta} \Delta \varphi_{b}}{-\theta_{b+1} \beta+e^{i \delta} \Delta \theta_{b}}
$$

Thus, condition (5.15) will be satisfied if we choose $v$ by (5.18) for pure imaginary values of $\beta$. On the other hand, when $\beta$ runs in (5.18) the imaginary axis, $v(\beta)$ describes a circle $C_{b}$ in the $v$-plane. The center of the circle is symmetric point of the point at infinity with respect to the circle. Since

$$
v\left(\beta^{\prime}\right)=\infty, \quad \text { where } \quad \beta^{\prime}=e^{i \delta} \frac{\Delta \theta_{b}}{\theta_{b+1}},
$$

the point

$$
\beta_{0}=-\overline{\beta^{\prime}}=-e^{-i \delta} \frac{\Delta \bar{\theta}_{b}}{\bar{\theta}_{b+1}}
$$

which is symmetric point of the point $\beta^{\prime}$ with respect to the imaginary axis of the $\beta$-plane, is mapped onto the center of $C_{b}$. So the center of $C_{b}$ is located at the point

$$
v\left(\beta_{0}\right)=-\frac{e^{-i \delta} \varphi_{b+1} \Delta \bar{\theta}_{b}+e^{i \delta}\left(\Delta \varphi_{b}\right) \bar{\theta}_{b+1}}{e^{-i \delta} \theta_{b+1} \Delta \bar{\theta}_{b}+e^{i \delta} \bar{\theta}_{b+1} \Delta \theta_{b}} .
$$

Note that the denominator in (5.19) is different from zero. This fact follows from the equality

$$
\begin{aligned}
e^{-i \delta} \theta_{b+1} \Delta \bar{\theta}_{b}+e^{i \delta} \bar{\theta}_{b+1} \Delta \theta_{b} & =2 \operatorname{Re}\left\{\left.e^{i \delta}\left(\Delta \theta_{n}\right) \bar{\theta}_{n+1}\right|_{1} ^{b}\right\} \\
& =(2 \cos \delta) \sum_{n=2}^{b}\left(\left|\Delta \theta_{n}\right|^{2}+q_{n}\left|\theta_{n}\right|^{2}\right),
\end{aligned}
$$

which can be derived as (5.13) taking into account (5.10). The radius $R_{b}$ of the circle $C_{b}$ is equal to the distance between the center $v\left(\beta_{0}\right)$ of $C_{b}$ and the point $v(0)$ on the circle. Calculating the difference $v\left(\beta_{0}\right)-v(0)$ by using (5.17)-(5.20) we easily find that

$$
R_{b}=\frac{1}{(2 \cos \delta) \sum_{n=2}^{b}\left(\left|\Delta \theta_{n}\right|^{2}+q_{n}\left|\theta_{n}\right|^{2}\right)} .
$$


Further, since

$$
\operatorname{Re}\left(e^{i \delta} \bar{\theta}_{b+1} \Delta \theta_{b}\right)=-\left|\theta_{b+1}\right|^{2} \operatorname{Re} \beta_{0},
$$

we get from $(5.20)$

$$
(\cos \delta) \sum_{n=2}^{b}\left(\left|\Delta \theta_{n}\right|^{2}+q_{n}\left|\theta_{n}\right|^{2}\right)=-\left|\theta_{b+1}\right|^{2} \operatorname{Re} \beta_{0}
$$

Therefore $\operatorname{Re} \beta_{0}<0$. This means that the left half-plane of the $\beta$-plane is mapped onto the interior of the circle $C_{b}$. Consequently, $v(\beta)$ belongs to the interior of the circle $C_{b}$ if and only if $\operatorname{Re} \beta<0$. This inequality is equivalent by (5.13), (5.16) to

$$
(\cos \delta) \sum_{n=2}^{b}\left(\left|\Delta \psi_{n}\right|^{2}+q_{n}\left|\psi_{n}\right|^{2}\right)<\operatorname{Re}\left(v e^{-i \delta}\right) .
$$

Thus, $v$ belongs to the interior of the circle $C_{b}$ if and only if the inequality (5.21) holds and $v$ lies on the circle $C_{b}$ if and only if

$$
(\cos \delta) \sum_{n=2}^{b}\left(\left|\Delta \psi_{n}\right|^{2}+q_{n}\left|\psi_{n}\right|^{2}\right)=\operatorname{Re}\left(v e^{-i \delta}\right) .
$$

Now let $b_{2}>b_{1}$. Then if $v$ is inside or on $C_{b_{2}}$

$$
(\cos \delta) \sum_{n=2}^{b_{1}}\left(\left|\Delta \psi_{n}\right|^{2}+q_{n}\left|\psi_{n}\right|^{2}\right)<(\cos \delta) \sum_{n=2}^{b_{2}}\left(\left|\Delta \psi_{n}\right|^{2}+q_{n}\left|\psi_{n}\right|^{2}\right) \leq \operatorname{Re}\left(v e^{-i \delta}\right)
$$

and therefore $v$ is inside $C_{b_{1}}$. This means $C_{b_{1}}$ contains $C_{b_{2}}$ in its interior if $b_{2}>b_{1}$. It follows that, as $b \rightarrow \infty$, the circles $C_{b}$ converge either to a limit-circle or to a limit-point. If $\widehat{v}$ is the limit-point or any point on the limit-circle, then $\widehat{v}$ is inside any $C_{b}$. Hence

$$
(\cos \delta) \sum_{n=2}^{b}\left(\left|\Delta \psi_{n}\right|^{2}+q_{n}\left|\psi_{n}\right|^{2}\right)<\operatorname{Re}\left(\widehat{v} e^{-i \delta}\right),
$$

where

$$
\psi_{n}=\varphi_{n}+\widehat{v} \theta_{n}, \quad n \in \mathbb{Z},
$$

and letting $b \rightarrow \infty$ we get

$$
(\cos \delta) \sum_{n=2}^{\infty}\left(\left|\Delta \psi_{n}\right|^{2}+q_{n}\left|\psi_{n}\right|^{2}\right) \leq \operatorname{Re}\left(\widehat{v} e^{-i \delta}\right) .
$$

It also follows that

$$
\operatorname{Re}\left(\widehat{v} e^{-i \delta}\right)>0
$$

Since $q_{n} \geq c>0$, (5.23) implies that for the solution $\psi=\left(\psi_{n}\right)$ defined by (5.22) we have (5.4). Thus, the statement of the theorem concerning the solution $\psi=\left(\psi_{n}\right)$ is proved.

Let us now show existence of the solution $\chi=\left(\chi_{n}\right)$ satisfying (5.4). We seek the desired solution $\theta=\left(\theta_{n}\right)$ of problem (5.1), (5.2) in the form

$$
\chi_{n}=\varphi_{n}+u \theta_{n}, \quad n \in \mathbb{Z},
$$


where $u$ is a complex constant to be determined.

Take an arbitrary integer $a \leq-1$. Applying (5.8) to the equations

$$
\begin{aligned}
& -\Delta^{2} \chi_{n-1}+q_{n} \chi_{n}=0, \quad n \in \mathbb{Z}_{0}, \\
& \chi_{-1}=\chi_{1}, \quad \Delta \chi_{-1}=e^{2 i \delta} \Delta \chi_{1},
\end{aligned}
$$

we get

$$
\sum_{n=a}^{-1}\left(\left|\Delta \chi_{n}\right|^{2}+q_{n}\left|\chi_{n}\right|^{2}\right)=\left.\left(\Delta \chi_{n}\right) \bar{\chi}_{n+1}\right|_{a-1} ^{-1}
$$

Since

$$
\Delta \chi_{-1}=-e^{2 i \delta}, \quad \chi_{0}=1-e^{2 i \delta}+u
$$

we have

$$
\sum_{n=a}^{-1}\left(\left|\Delta \chi_{n}\right|^{2}+q_{n}\left|\chi_{n}\right|^{2}\right)=-e^{2 i \delta}+1-\bar{u} e^{2 i \delta}-\left(\Delta \chi_{a-1}\right) \bar{\chi}_{a} .
$$

Multiply both sides of (5.25) by $e^{-i \delta}$ and take then the real part of both sides to get

$$
(\cos \delta) \sum_{n=a}^{-1}\left(\left|\Delta \chi_{n}\right|^{2}+q_{n}\left|\chi_{n}\right|^{2}\right)=-\operatorname{Re}\left(u e^{-i \delta}\right)-\operatorname{Re}\left\{e^{-i \delta}\left(\Delta \chi_{a-1}\right) \bar{\chi}_{a}\right\} .
$$

Now we choose $u$ so that to have

$$
\operatorname{Re}\left\{e^{-i \delta}\left(\Delta \chi_{a-1}\right) \bar{\chi}_{a}\right\}=0 .
$$

Since

$$
\operatorname{Re}\left\{e^{-i \delta}\left(\Delta \chi_{a-1}\right) \bar{\chi}_{a}\right\}=\left|\chi_{a}\right|^{2} \operatorname{Re}\left\{e^{-i \delta} \frac{\Delta \chi_{a-1}}{\chi_{a}}\right\},
$$

it is sufficient for (5.27) to have

$$
\operatorname{Re}\left\{e^{-i \delta} \frac{\Delta \chi_{a-1}}{\chi_{a}}\right\}=0
$$

Then (5.26) becomes

$$
(\cos \delta) \sum_{n=a}^{-1}\left(\left|\Delta \chi_{n}\right|^{2}+q_{n}\left|\chi_{n}\right|^{2}\right)=-\operatorname{Re}\left(u e^{-i \delta}\right) .
$$

The condition (5.28) can be written as

$$
e^{-i \delta} \frac{\Delta \varphi_{a-1}+u \Delta \theta_{a-1}}{\varphi_{a}+u \theta_{a}}=\alpha,
$$

where $\alpha$ is a pure imaginary number $(\alpha=i t, t \in \mathbb{R})$. Note that

$$
\varphi_{a} \Delta \theta_{a-1}-\left(\Delta \varphi_{a-1}\right) \theta_{a}=W_{a-1}(\varphi, \theta)=e^{2 i \delta} \neq 0
$$


by (5.11). Therefore (5.29) defines a linear-fractional transformation of the complex $u$-plane onto the complex $\alpha$-plane. Solving (5.29) for $u$, we get

$$
u(\alpha)=\frac{\varphi_{a} \alpha-e^{-i \delta} \Delta \varphi_{a-1}}{-\theta_{a} \alpha+e^{-i \delta} \Delta \theta_{a-1}} .
$$

Thus, condition (5.28) will be satisfied if we choose $u$ by (5.31) for pure imaginary values of $\alpha$. On the other hand, when $\alpha$ runs in (5.31) the imaginary axis, $u(\alpha)$ describes a circle $K_{a}$ in the $u$-plane. The center of the circle is symmetric point of the point at infinity with respect to the circle. Since

$$
u\left(\alpha^{\prime}\right)=\infty, \quad \text { where } \quad \alpha^{\prime}=e^{-i \delta} \frac{\Delta \theta_{a-1}}{\theta_{a}},
$$

the point

$$
\alpha_{0}=-\overline{\alpha^{\prime}}=-e^{i \delta} \frac{\Delta \bar{\theta}_{a-1}}{\bar{\theta}_{a}}
$$

which is symmetric point of the point $\alpha^{\prime}$ with respect to the imaginary axis of the $\alpha$-plane, is mapped onto the center of $K_{a}$. So the center of $K_{a}$ is located at the point

$$
u\left(\alpha_{0}\right)=-\frac{e^{i \delta} \varphi_{a} \Delta \bar{\theta}_{a-1}+e^{-i \delta}\left(\Delta \varphi_{a-1}\right) \bar{\theta}_{a}}{e^{i \delta} \theta_{a} \Delta \bar{\theta}_{a-1}+e^{-i \delta} \bar{\theta}_{a} \Delta \theta_{a-1}} .
$$

Note that the denominator in (5.32) is different from zero. This fact follows from the equality

$$
\begin{gathered}
e^{-i \delta} \theta_{a} \Delta \bar{\theta}_{a-1}+e^{i \delta} \bar{\theta}_{a} \Delta \theta_{a-1}=2 \operatorname{Re}\left\{e^{i \delta}\left(\Delta \theta_{a-1}\right) \bar{\theta}_{a}\right\} \\
=-(2 \cos \delta) \sum_{n=a}^{-1}\left(\left|\Delta \theta_{n}\right|^{2}+q_{n}\left|\theta_{n}\right|^{2}\right)
\end{gathered}
$$

which can be derived as (5.26) taking into account $\Delta \theta_{-1}=0, \theta_{0}=1$. Calculating the difference $u\left(\alpha_{0}\right)-u(0)$ we easily find the radius $R_{a}=\left|u\left(\alpha_{0}\right)-u(0)\right|$ of the circle $K_{a}$, using (5.30)-(5.33),

$$
R_{a}=\frac{1}{(2 \cos \delta) \sum_{n=a}^{-1}\left(\left|\Delta \theta_{n}\right|^{2}+q_{n}\left|\theta_{n}\right|^{2}\right)} .
$$

Further, since

$$
\operatorname{Re}\left(e^{-i \delta} \bar{\theta}_{a} \Delta \theta_{a-1}\right)=-\left|\theta_{a}\right|^{2} \operatorname{Re} \alpha_{0},
$$

we get from (5.33)

$$
(\cos \delta) \sum_{n=a}^{-1}\left(\left|\Delta \theta_{n}\right|^{2}+q_{n}\left|\theta_{n}\right|^{2}\right)=\left|\theta_{a}\right|^{2} \operatorname{Re} \alpha_{0} .
$$

Therefore $\operatorname{Re} \alpha_{0}>0$. This means that the right half-plane of the $\alpha$-plane is mapped onto the interior of the circle $K_{a}$. Consequently, $u(\alpha)$ lies inside the circle $K_{a}$ if and only if $\operatorname{Re} \alpha>0$. This inequality is equivalent by (5.26), (5.29) to

$$
(\cos \delta) \sum_{n=a}^{-1}\left(\left|\Delta \chi_{n}\right|^{2}+q_{n}\left|\chi_{n}\right|^{2}\right)<-\operatorname{Re}\left(u e^{-i \delta}\right) .
$$


Thus, $u$ lies inside the circle $K_{a}$ if and only if the inequality (5.34) holds and $u$ lies on the circle $K_{a}$ if and only if

$$
(\cos \delta) \sum_{n=a}^{-1}\left(\left|\Delta \chi_{n}\right|^{2}+q_{n}\left|\chi_{n}\right|^{2}\right)=-\operatorname{Re}\left(u e^{-i \delta}\right) .
$$

Now let $a_{2}<a_{1}$. Then if $u$ is inside or on $K_{a_{2}}$

$$
(\cos \delta) \sum_{n=a_{1}}^{-1}\left(\left|\Delta \chi_{n}\right|^{2}+q_{n}\left|\chi_{n}\right|^{2}\right)<(\cos \delta) \sum_{n=a_{2}}^{-1}\left(\left|\Delta \chi_{n}\right|^{2}+q_{n}\left|\chi_{n}\right|^{2}\right) \leq-\operatorname{Re}\left(u e^{-i \delta}\right)
$$

and therefore $u$ is inside $K_{a_{1}}$. This means $K_{a_{1}}$ contains $K_{a_{2}}$ in its interior if $a_{2}<a_{1}$. It follows that, as $a \rightarrow-\infty$, the circles $K_{a}$ converge either to a limit-circle or to a limit-point. If $\widehat{u}$ is the limit-point or any point on the limit-circle, then $\widehat{u}$ is inside any $K_{a}$. Hence

$$
(\cos \delta) \sum_{n=a}^{-1}\left(\left|\Delta \chi_{n}\right|^{2}+q_{n}\left|\chi_{n}\right|^{2}\right)<-\operatorname{Re}\left(\widehat{u} e^{-i \delta}\right),
$$

where

$$
\chi_{n}=\varphi_{n}+\widehat{u} \theta_{n}, \quad n \in \mathbb{Z},
$$

and letting $a \rightarrow-\infty$ we get

$$
(\cos \delta) \sum_{-\infty}^{n=-1}\left(\left|\Delta \chi_{n}\right|^{2}+q_{n}\left|\chi_{n}\right|^{2}\right) \leq-\operatorname{Re}\left(\widehat{u} e^{-i \delta}\right) .
$$

It also follows that

$$
\operatorname{Re}\left(\widehat{u} e^{-i \delta}\right)<0 .
$$

Since $q_{n} \geq c>0$, (5.36) implies that for the solution $\chi=\left(\chi_{n}\right)$ defined by (5.35) we have (5.4). Thus, the statement of the theorem concerning the solution $\chi=\left(\chi_{n}\right)$ is also proved.

Finally, let us show that the solutions $\psi=\left(\psi_{n}\right)$ and $\chi=\left(\chi_{n}\right)$ defined by (5.22) and (5.35), respectively, are linearly independent. We have

$$
W_{n}(\psi, \chi)=W_{n}(\varphi+\widehat{v} \theta, \varphi+\widehat{u} \theta)=(\widehat{u}-\widehat{v}) W_{n}(\varphi, \theta) .
$$

Next, $W_{n}(\varphi, \theta) \neq 0$ by (5.11) and $\widehat{u} \neq \widehat{v}$ by (5.24), (5.37). Therefore $W_{n}(\psi, \chi) \neq 0$ and hence $\psi$ and $\chi$ are linearly independent by Theorem 5 .

\section{The inverse operator $L^{-1}$}

The following lemma will play crucial role in this and next sections.

Lemma 2. Let us set

$$
\sigma_{n}= \begin{cases}e^{-i \delta} & \text { for } \quad n \leq-1 \\ e^{i \delta} & \text { for } n \geq 0\end{cases}
$$

Under the conditions of Lemma 1 the following formula holds:

$$
\begin{aligned}
(\cos \delta) & \left(\sum_{n=a}^{-1}+\sum_{n=2}^{b}\right)\left(\left|\Delta y_{n}\right|^{2}+q_{n}\left|y_{n}\right|^{2}\right) \\
= & \operatorname{Re}\left\{\left.\sigma_{n}\left(\Delta y_{n}\right) \bar{y}_{n+1}\right|_{a-1} ^{b}+\left(\sum_{n=a}^{-1}+\sum_{n=2}^{b}\right) \sigma_{n} f_{n} \bar{y}_{n}\right\} .
\end{aligned}
$$


Proof. To prove (6.2) we multiply (5.8) by $e^{-i \delta}$ and (5.7) by $e^{i \delta}$ and add together to get

$$
\begin{aligned}
& \left(e^{-i \delta} \sum_{n=a}^{-1}+e^{i \delta} \sum_{n=2}^{b}\right)\left(\left|\Delta y_{n}\right|^{2}+q_{n}\left|y_{n}\right|^{2}\right) \\
& \quad=\left.e^{-i \delta}\left(\Delta y_{n}\right) \bar{y}_{n+1}\right|_{a-1} ^{-1}+\left.e^{i \delta}\left(\Delta y_{n}\right) \bar{y}_{n+1}\right|_{1} ^{b}+\left(e^{-i \delta} \sum_{n=a}^{-1}+e^{i \delta} \sum_{n=2}^{b}\right) f_{n} \bar{y}_{n} .
\end{aligned}
$$

Next, using the conditions (5.6) we have

$$
\begin{aligned}
e^{-i \delta} & \left(\Delta y_{-1}\right) \bar{y}_{0}-e^{i \delta}\left(\Delta y_{1}\right) \bar{y}_{2}=e^{i \delta}\left(\Delta y_{1}\right) \bar{y}_{0}-e^{i \delta}\left(\Delta y_{1}\right) \bar{y}_{2} \\
& =e^{i \delta}\left(\Delta y_{1}\right)\left(\bar{y}_{0}-\bar{y}_{2}\right)=e^{i \delta}\left(\Delta y_{1}\right)\left(\bar{y}_{0}-\bar{y}_{1}+\bar{y}_{1}-\bar{y}_{2}\right) \\
& =e^{i \delta}\left(\Delta y_{1}\right)\left(\bar{y}_{0}-\bar{y}_{-1}+\bar{y}_{1}-\bar{y}_{2}\right)=e^{i \delta}\left(\Delta y_{1}\right)\left(\overline{\Delta y_{-1}}-\overline{\Delta y_{1}}\right) \\
& =-e^{i \delta}\left|\Delta y_{1}\right|^{2}+e^{i \delta}\left(\Delta y_{1}\right) e^{-2 i \delta} \overline{\Delta y_{1}}=-e^{i \delta}\left|\Delta y_{1}\right|^{2}+e^{-i \delta}\left|\Delta y_{1}\right|^{2}=-2 i(\sin \delta)\left|\Delta y_{1}\right|^{2} .
\end{aligned}
$$

Therefore taking (6.1) into account we can rewrite (6.3) in the form

$$
\begin{aligned}
& \left(\sum_{n=a}^{-1}+\sum_{n=2}^{b}\right) \sigma_{n}\left(\left|\Delta y_{n}\right|^{2}+q_{n}\left|y_{n}\right|^{2}\right) \\
& \quad=-2 i(\sin \delta)\left|\Delta y_{1}\right|^{2}+\left.\sigma_{n}\left(\Delta y_{n}\right) \bar{y}_{n+1}\right|_{a-1} ^{b}+\left(\sum_{n=a}^{-1}+\sum_{n=2}^{b}\right) \sigma_{n} f_{n} \bar{y}_{n} .
\end{aligned}
$$

Taking in (6.4) the real parts of both sides and taking into account that $\operatorname{Re} \sigma_{n}=\cos \delta$ for all $n$, we obtain (6.2).

Let $L: D \subset l_{0}^{2} \rightarrow l_{0}^{2}$ be the operator defined above in Section 2. Further, let $\psi=\left(\psi_{n}\right)$ and $\chi=\left(\chi_{n}\right)$, where $n \in \mathbb{Z}$, be solutions of problem (5.1), (5.2), constructed in Theorem 8. Let us introduce the discrete Green function

$$
G_{n k}=\frac{1}{W_{k}(\psi, \chi)} \begin{cases}\chi_{k} \psi_{n} & \text { if } \quad k \leq n, \\ \chi_{n} \psi_{k} & \text { if } \quad k \geq n,\end{cases}
$$

of discrete variables $k, n \in \mathbb{Z}$. Note that by (5.38) and (5.11), we have

$$
W_{0}(\psi, \chi)=-(\widehat{u}-\widehat{v}) e^{2 i \delta}, \quad W_{k}(\psi, \chi)= \begin{cases}(\widehat{u}-\widehat{v}) e^{2 i \delta} & \text { if } \quad k \leq-1 \\ \widehat{u}-\widehat{v} & \text { if } \quad k \geq 1\end{cases}
$$

and, besides,

$$
\widehat{u} \neq \widehat{v}
$$

by (5.24) and (5.37).

Theorem 9. Under the condition (5.3) the inverse operator $L^{-1}$ exists and is a bounded operator defined on the whole space $l_{0}^{2}$. Next, for every $f=\left(f_{n}\right) \in l_{0}^{2}$

$$
\left(L^{-1} f\right)_{n}=\sum_{k \in \mathbb{Z}_{0}} G_{n k} f_{k}, \quad n \in \mathbb{Z}_{0}
$$

and

$$
\left\|L^{-1} f\right\| \leq \frac{1}{c \cos \delta}\|f\| \quad \text { for all } f \in l_{0}^{2},
$$

where $c$ is a constant from condition (5.3) and $\delta$ is from (5.2), $\|\cdot\|$ denotes the norm of space $l_{0}^{2}$. 
Proof. Let us show that

$\operatorname{ker} L=\{y \in D: L y=0\}$

consists only of the zero element. Indeed, if $y \in D$ and $L y=0$, then $(y)_{n \in \mathbb{Z}_{0}}$ satisfies the equation

$$
-\Delta^{2} y_{n-1}+q_{n} y_{n}=0, \quad n \in \mathbb{Z}_{0},
$$

in which $y_{0}$ and $y_{1}$ (these values arise in (6.8) for $n=-1$ and $n=2$, respectively) are defined from the equations

$$
y_{-1}=y_{1}, \quad \Delta y_{-1}=e^{2 i \delta} \Delta y_{1} .
$$

Since $\chi$ and $\psi$ form a fundamental system of solutions of (6.8), (6.9), we can write

$$
y_{n}=C_{1} \psi_{n}+C_{2} \chi_{n}, \quad n \in \mathbb{Z},
$$

with some constants $C_{1}$ and $C_{2}$. Hence

$$
W_{n}(y, \psi)=C_{1} W_{n}(\psi, \psi)+C_{2} W_{n}(\chi, \psi), \quad n \in \mathbb{Z} .
$$

Next, since $y \in l_{0}^{2}$, we have $y_{n} \rightarrow 0$ as $|n| \rightarrow \infty$ and by (5.4) we have $\psi_{n} \rightarrow 0$ as $n \rightarrow \infty$ Hence $W_{n}(y, \psi) \rightarrow 0$ as $n \rightarrow \infty$. Besides $W_{n}(\psi, \psi)=0$ for all $n$ and $W_{n}(\chi, \psi)$ is equal to a nonzero constant for $n \geq 1$ by (6.5). Therefore taking the limit in (6.10) as $n \rightarrow \infty$ we get that $C_{2}=0$. It can similarly be shown, by considering $W_{n}(y, \chi)$, that $C_{1}=0$. Thus $y=0$.

It follows that the inverse operator $L^{-1}$ exists. Now take an arbitrary $f=\left(f_{n}\right)_{n \in \mathbb{Z}_{0}} \in l_{0}^{2}$ and extend the sequence $\left(f_{n}\right)_{n \in \mathbb{Z}_{0}}$ to the values $n=0$ and $n=1$ by setting

$$
f_{0}=f_{1}=0 \text {. }
$$

Let us put

$$
g_{n}=\sum_{k \in \mathbb{Z}_{0}} G_{n k} f_{k}=\sum_{k \in \mathbb{Z}} G_{n k} f_{k}=\psi_{n} \sum_{-\infty}^{k=n} \frac{\chi_{k} f_{k}}{W_{k}(\psi, \chi)}+\chi_{n} \sum_{k=n+1}^{\infty} \frac{\psi_{k} f_{k}}{W_{k}(\psi, \chi)}, \quad n \in \mathbb{Z} .
$$

Then it is easy to check that this sequence $\left(g_{n}\right)$, where $n \in \mathbb{Z}$, satisfies the equations

$$
\begin{aligned}
& -\Delta^{2} g_{n-1}+q_{n} g_{n}=f_{n}, \quad n \in \mathbb{Z}_{0}, \\
& g_{-1}=g_{1}, \quad \Delta g_{-1}=e^{2 i \delta} \Delta g_{1} .
\end{aligned}
$$

We want to show that $g=\left(g_{n}\right)_{n \in \mathbb{Z}_{0}} \in l_{0}^{2}$ and that

$$
\|g\| \leq \frac{1}{c \cos \delta}\|f\|
$$

For this purpose we take the sequences of integers $a_{m}$ and $b_{m}$ defined for any positive integer $m$, such that

$$
a_{m}<0<b_{m} \quad \text { and } \quad a_{m} \rightarrow-\infty, \quad b_{m} \rightarrow \infty \quad \text { as } \quad m \rightarrow \infty .
$$

Next, for each $m$ we define the sequence $\left(f_{n}^{(m)}\right)_{n \in \mathbb{Z}}$ by

$$
\begin{aligned}
& f_{n}^{(m)}=f_{n} \quad \text { if } \quad a_{m} \leq n \leq b_{m}, \\
& f_{n}^{(m)}=0 \quad \text { if } \quad n<a_{m} \quad \text { or } \quad n>b_{m},
\end{aligned}
$$


and put

$$
g_{n}^{(m)}=\sum_{k \in \mathbb{Z}_{0}} G_{n k} f_{k}^{(m)}=\sum_{k \in \mathbb{Z}} G_{n k} f_{k}^{(m)}=\psi_{n} \sum_{-\infty}^{k=n} \frac{\chi_{k} f_{k}^{(m)}}{W_{k}(\psi, \chi)}+\chi_{n} \sum_{k=n+1}^{\infty} \frac{\psi_{k} f_{k}^{(m)}}{W_{k}(\psi, \chi)}, \quad n \in \mathbb{Z} .
$$

It follows that

$$
g_{n}^{(m)}=\left\{\begin{array}{c}
\chi_{n} \sum_{k=a_{m}}^{b_{m}} \frac{\psi_{k} f_{k}}{W_{k}(\psi, \chi)} \quad \text { if } n<a_{m}, \\
\psi_{n} \sum_{k=a_{m}}^{b_{m}} \frac{\chi_{k} f_{k}}{W_{k}(\psi, \chi)} \quad \text { if } n>b_{m} .
\end{array}\right.
$$

We have also that, for each $m$,

$$
\begin{aligned}
& -\Delta^{2} g_{n-1}^{(m)}+q_{n} g_{n}^{(m)}=f_{n}^{(m)}, \quad n \in \mathbb{Z}_{0}, \\
& g_{-1}^{(m)}=g_{1}^{(m)}, \quad \Delta g_{-1}^{(m)}=e^{2 i \delta} \Delta g_{1}^{(m)} .
\end{aligned}
$$

Fix $m$ and take a positive integer $N$ such that

$$
-N<a_{m} \quad \text { and } \quad b_{m}<N .
$$

Then applying Lemma 2 to (6.18), (6.19) we can write

$$
\begin{aligned}
& (\cos \delta)\left(\sum_{n=-N}^{-1}+\sum_{n=2}^{N}\right)\left(\left|\Delta g_{n}^{(m)}\right|^{2}+q_{n}\left|g_{n}^{(m)}\right|^{2}\right) \\
& =\operatorname{Re}\left\{\left.\sigma_{n}\left(\Delta g_{n}^{(m)}\right) \bar{g}_{n+1}^{(m)}\right|_{-N-1} ^{N}+\left(\sum_{n=-N}^{-1}+\sum_{n=2}^{N}\right) \sigma_{n} f_{n}^{(m)} \bar{g}_{n}^{(m)}\right\} .
\end{aligned}
$$

It follows from (6.17) by (5.4) that

$$
\sum_{n \in \mathbb{Z}}\left|g_{n}^{(m)}\right|^{2}<\infty
$$

Therefore the sums on the right-hand side of (6.20) are convergent as $N \rightarrow \infty$ and besides

$$
\operatorname{Re}\left\{\left.\sigma_{n}\left(\Delta g_{n}^{(m)}\right) \bar{g}_{n+1}^{(m)}\right|_{-N-1} ^{N}\right\} \rightarrow 0 \quad \text { as } \quad N \rightarrow \infty .
$$

(note that $\left|\sigma_{n}\right|=1$ for all $n$ by (6.1)). Now taking the limit in (6.20) as $N \rightarrow \infty$, we get

$$
(\cos \delta) \sum_{n \in \mathbb{Z}_{0}}\left(\left|\Delta g_{n}^{(m)}\right|^{2}+q_{n}\left|g_{n}^{(m)}\right|^{2}\right)=\operatorname{Re} \sum_{n \in \mathbb{Z}_{0}} \sigma_{n} f_{n}^{(m)} \bar{g}_{n}^{(m)} .
$$

Using the condition (5.3) we get from (6.21) that

$$
\begin{aligned}
& \sum_{n \in \mathbb{Z}_{0}}\left|g_{n}^{(m)}\right|^{2} \leq \frac{1}{c \cos \delta} \operatorname{Re} \sum_{n \in \mathbb{Z}_{0}} \sigma_{n} f_{n}^{(m)} \bar{g}_{n}^{(m)} \leq \frac{1}{c \cos \delta}\left|\sum_{n \in \mathbb{Z}_{0}} \sigma_{n} f_{n}^{(m)} \bar{g}_{n}^{(m)}\right| \\
& \quad \leq \frac{1}{c \cos \delta} \sum_{n \in \mathbb{Z}_{0}}\left|f_{n}^{(m)} \bar{g}_{n}^{(m)}\right| \leq \frac{1}{c \cos \delta}\left\{\sum_{n \in \mathbb{Z}_{0}}\left|f_{n}^{(m)}\right|^{2}\right\}^{1 / 2}\left\{\sum_{n \in \mathbb{Z}_{0}}\left|g_{n}^{(m)}\right|^{2}\right\}^{1 / 2} .
\end{aligned}
$$


Hence

$$
\left\{\sum_{n \in \mathbb{Z}_{0}}\left|g_{n}^{(m)}\right|^{2}\right\}^{1 / 2} \leq \frac{1}{c \cos \delta}\left\{\sum_{n \in \mathbb{Z}_{0}}\left|f_{n}^{(m)}\right|^{2}\right\}^{1 / 2},
$$

that is,

$$
\left\|g^{(m)}\right\| \leq \frac{1}{c \cos \delta}\left\|f^{(m)}\right\| .
$$

Writing (6.18), (6.19) for $m=m_{1}$ and $m=m_{2}$, and subtracting the obtained equations side-by-side, we get

$$
\begin{aligned}
& -\Delta^{2}\left[g_{n-1}^{\left(m_{1}\right)}-g_{n-1}^{\left(m_{2}\right)}\right]+q_{n}\left[g_{n}^{\left(m_{1}\right)}-g_{n}^{\left(m_{2}\right)}\right]=f_{n}^{\left(m_{1}\right)}-f_{n}^{\left(m_{2}\right)}, \quad n \in \mathbb{Z}_{0}, \\
& g_{-1}^{\left(m_{1}\right)}-g_{-1}^{\left(m_{2}\right)}=g_{1}^{\left(m_{1}\right)}-g_{1}^{\left(m_{2}\right)}, \quad \Delta\left[g_{-1}^{\left(m_{1}\right)}-g_{-1}^{\left(m_{2}\right)}\right]=e^{2 i \delta} \Delta\left[g_{1}^{\left(m_{1}\right)}-g_{1}^{\left(m_{2}\right)}\right] .
\end{aligned}
$$

Hence, repeating the same reasonings as above, we get

$$
\left\|g^{\left(m_{1}\right)}-g^{\left(m_{2}\right)}\right\| \leq \frac{1}{c \cos \delta}\left\|f^{\left(m_{1}\right)}-f^{\left(m_{2}\right)}\right\| .
$$

It follows that $g^{(m)}$ converges in $l_{0}^{2}$ to an element $\widetilde{g}$ as $m \rightarrow \infty$. On the other hand, it can be seen from (6.11), (6.17) taking into account (6.15), (6.16) that

$$
g_{n}^{(m)} \rightarrow g_{n} \quad \text { as } m \rightarrow \infty,
$$

for each $n$. Consequently, $\widetilde{g}=g$ and hence $g \in l_{0}^{2}$. Passing in (6.22) to the limit as $m \rightarrow \infty$, we get (6.14).

Next, from (6.12) we have

$$
q_{n} g_{n}=f_{n}+g_{n-1}-2 g_{n}+g_{n+1}, \quad n \in \mathbb{Z}_{0} .
$$

Hence $\left(q_{n} g_{n}\right)_{n \in \mathbb{Z}_{0}} \in l_{0}^{2}$. Therefore $g \in D$, where $D$ is the domain of the operator $L$. If we define an operator $B: l_{0}^{2} \rightarrow l_{0}^{2}$ by the formula $B f=g$, where $f=\left(f_{n}\right)_{n \in \mathbb{Z}_{0}} \in l_{0}^{2}$ and $g=\left(g_{n}\right)_{n \in \mathbb{Z}_{0}}$ with $g_{n}$ defined by (6.11), then we get by (6.12), (6.13) that $L B f=f$. Therefore $B$ is the inverse of the operator $L: B=L^{-1}$, so that $g=L^{-1} f$ and from (6.11) and (6.14) we get (6.6) and (6.7), respectively.

\section{Completely continuity of the operator $L^{-1}$}

In this section we will show that the operator $L^{-1}$ is completely continuous, that is, it is continuous and maps bounded sets into relatively compact sets.

Theorem 10. Let

$$
q_{n} \geq c>0 \quad \text { for } n \in \mathbb{Z}_{0}
$$

and

$$
\lim _{|n| \rightarrow \infty} q_{n}=\infty
$$

Then the operator $L^{-1}$ is completely continuous. 
Proof. The operator $L^{-1}$ is continuous in virtue of (6.7) that holds under the condition (7.1). In order to show that $L^{-1}$ maps bounded sets into relatively compact sets consider any bounded set $X$ in $l_{0}^{2}$,

$$
X=\left\{f \in l_{0}^{2}:\|f\| \leq d\right\},
$$

and prove that $L^{-1}(X)=Y$ is relatively compact in $l_{0}^{2}$. To this end, we use the following known (see [17]) criterion for the relative compactness in $l_{0}^{2}: A$ set $Y \subset l_{0}^{2}$ is relatively compact if and only if $Y$ is bounded and for every $\varepsilon>0$ there exists a positive integer $n_{0}$ (depending only on $\varepsilon$ ) such that

$$
\sum_{|n|>n_{0}}\left|y_{n}\right|^{2} \leq \varepsilon \quad \text { for all } y \in Y
$$

Take an arbitrary $f \in X$ and set

$$
L^{-1} f=y \text {. }
$$

Then $L y=f$ or explicitly

$$
-\Delta^{2} y_{n-1}+q_{n} y_{n}=f_{n}, \quad n \in \mathbb{Z}_{0},
$$

where $y_{0}$ and $y_{1}$ are defined from the equations

$$
y_{-1}=y_{1}, \quad \Delta y_{-1}=e^{2 i \delta} \Delta y_{1} .
$$

Note that $y_{0}$ and $y_{1}$ are needed when we write out equation (7.3) for $n=-1$ and $n=2$, respectively.

Applying Lemma 2 to (7.3), (7.4), we get that for any integers $a \leq-1$ and $b \geq 2$,

$$
\begin{aligned}
(\cos \delta) & \left(\sum_{n=a}^{-1}+\sum_{n=2}^{b}\right)\left(\left|\Delta y_{n}\right|^{2}+q_{n}\left|y_{n}\right|^{2}\right) \\
= & \operatorname{Re}\left\{\left.\sigma_{n}\left(\Delta y_{n}\right) \bar{y}_{n+1}\right|_{a-1} ^{b}+\left(\sum_{n=a}^{-1}+\sum_{n=2}^{b}\right) \sigma_{n} f_{n} \bar{y}_{n}\right\},
\end{aligned}
$$

where $\sigma_{n}$ is defined by $(6.1)$.

Since $f, y \in l_{0}^{2}$ and $\left|\sigma_{n}\right|=1$, the sums on the right-hand side of (7.5) are convergent as $a \rightarrow-\infty, b \rightarrow \infty$. Also from $y \in l_{0}^{2}$ it follows that $y_{n} \rightarrow 0$ as $|n| \rightarrow \infty$ so that

$$
\left.\sigma_{n}\left(\Delta y_{n}\right) \bar{y}_{n+1}\right|_{a-1} ^{b} \rightarrow 0 \quad \text { as } \quad a \rightarrow-\infty, \quad b \rightarrow \infty .
$$

Consequently, we arrive at the equality

$$
(\cos \delta) \sum_{n \in \mathbb{Z}_{0}}\left(\left|\Delta y_{n}\right|^{2}+q_{n}\left|y_{n}\right|^{2}\right)=\operatorname{Re} \sum_{n \in \mathbb{Z}_{0}} \sigma_{n} f_{n} \bar{y}_{n} .
$$

Hence

$$
(\cos \delta) \sum_{n \in \mathbb{Z}_{0}} q_{n}\left|y_{n}\right|^{2} \leq \operatorname{Re} \sum_{n \in \mathbb{Z}_{0}} \sigma_{n} f_{n} \bar{y}_{n}
$$

and therefore using (7.1) and $\|f\| \leq d$, we get

$$
\|y\| \leq \frac{d}{c \cos \delta} \quad \text { for all } y \in Y .
$$

This means that the set $Y=L^{-1}(X)$ is bounded. 
From (7.6) we also have, using (7.7),

$$
\sum_{n \in \mathbb{Z}_{0}} q_{n}\left|y_{n}\right|^{2} \leq \frac{d^{2}}{c \cos ^{2} \delta} \quad \text { for all } y \in Y .
$$

Take now an arbitrary $\varepsilon>0$. By condition (7.2) we can choose a positive integer $n_{0}$ such that

$$
q_{n} \geq \frac{d^{2}}{\varepsilon c \cos ^{2} \delta} \quad \text { for }|n|>n_{0} .
$$

Then we get from (7.8) that

$$
\sum_{|n|>n_{0}}\left|y_{n}\right|^{2} \leq \varepsilon \quad \text { for all } y \in Y \text {. }
$$

Thus the completely continuity of the operator $L^{-1}$ is proved.

Corollary 2. The operator $A=M^{-1} L$ is invertible and its inverse $A^{-1}=L^{-1} M$ is a completely continuous operator to be a product of completely continuous operator with bounded operator. Therefore the spectrum of the operator $A$ is discrete.

\section{Conclusions}

In this paper we have explored a new class of discrete non-Hermitian quantum systems. The concept of the spectrum for the considered discrete system is introduced and discreteness of the spectrum is proved under some simple conditions.

As a tool for the investigation we have established main statements for second order linear difference equations with impulse conditions (transition conditions). We have chosen a suitable (infinite-dimensional) Hilbert space and defined the main linear operator $A$ so that the spectrum of the problem in question coincides with the spectrum of $A$. Next, we have constructed the inverse $A^{-1}$ of the operator $A$ by using an appropriate discrete Green function. Finally, we have shown that the inverse operator $A^{-1}$ is completely continuous. This implies, in particular, discreteness of the spectrum of $A$.

\section{Acknowledgements}

This work was supported by Grant 106T549 from the Scientific and Technological Research Council of Turkey (TUBITAK). The author thanks Mesude Saglam and Gusein Guseinov for useful discussions.

\section{References}

[1] Bender C.M., Making sense of non-Hermitian Hamiltonians, Rep. Progr. Phys. 70 (2007), 947-1018, hep-th/0703096.

[2] Bender C.M., Boettcher S., Real spectra in non-Hermitian Hamiltonians having PT-symmetry, Phys. Rev. Lett. 80 (1998), 5243-5246, physics/9712001.

[3] Dorey P., Dunning C., Tateo T., Spectral equivalences, Bethe ansatz equations, and reality properties in PT-symmetric quantum mechanics, J. Phys. A: Math. Gen. 34 (2001), 5679-5704, hep-th/0103051.

[4] Shin K.C., On the reality of the eigenvalues for a class of PT-symmetric oscillators, Comm. Math. Phys. 229 (2002), 543-564, math-ph/0201013.

[5] Mostafazadeh A., Pseudo-Hermitian description of PT-symmetric systems defined on a complex contour, J. Phys. A: Math. Gen. 38 (2005), 3213-3234, quant-ph/0410012. 
[6] Kelley W.G., Peterson A.C., Difference equations. An introduction with applications, Academic Press, Inc., Boston, MA, 1991.

[7] Teschl G., Jacobi operators and completely integrable nonlinear lattices, Mathematical Surveys and Monographs, Vol. 72, American Mathematical Society, Providence, RI, 2000.

[8] Bender C.M., Meisinger P.N., Wang Q., Finite dimensional PT-symmetric Hamiltonians, J. Phys. A: Math. Gen. 36 (2003), 6791-6797, quant-ph/0303174.

[9] Weigret S., How to test for digonalizability: the discretized PT-invariant square-well potential, Czechoslovak J. Phys. 55 (2005), 1183-1186.

[10] Znojil M., Matching method and exact solvability of discrete PT-symmetric square wells, J. Phys. A: Math. Gen. 39 (2006), 10247-10261, quant-ph/0605209.

[11] Znojil M., Maximal couplings in PT-symmetric chain models with the real spectrum of energies, J. Phys. A: Math. Theor. 40 (2007), 4863-4875, math-ph/0703070.

[12] Znojil M., Tridiagonal $P T$-symmetric $N$ by $N$ Hamiltonians and fine-tuning of their obsevability domains in the strongly non-Hermitian regime, J. Phys. A: Math. Theor. 40 (2007), 13131-13148, arXiv:0709.1569.

[13] Jones H.F., Scattering from localized non-Hermitian potentials, Phys. Rev. D 76 (2007), 125003, 5 pages, arXiv:0707.3031.

[14] Znojil M., Scattering theory with localized non-Hermiticities, Phys. Rev. D 78 (2008), 025026, 10 pages, arXiv:0805.2800.

[15] Ergun E., On the reality of the spectrum of a non-Hermitian discrete Hamiltonian, Rep. Math. Phys. 63 (2009), 75-93.

[16] Akhiezer N.I., Glazman I.M., Theory of linear operators in Hilbert space, Vol. 1, Ungar, New York, 1961.

[17] Lusternik L.A., Sobolev V.J., Elements of functional analysis, H. Ward Crowley Frederick Ungar Publishing Co., New York, 1961. 